\title{
ESTRATEGIAS DIDÁCTICAS PARA EL DESARROLLO \\ DE COMPETENCIAS Y PENSAMIENTO COMPLEJO \\ EN ESTUDIANTES UNIVERSITARIOS
}

\section{Didactic strategies for the development of competences and complex thinking in university students}

\author{
Dulio Oseda GAGO \\ Universidad Nacional de Cañete, Lima-Perú \\ doseda@undc.edu.pe \\ Código Orcid: https://orcid.org/0000-0002-3136-6094 \\ Ruth Katherine Mendivel Geronimo ${ }^{* *}$ \\ Universidad Nacional de Cañete, Lima-Perú \\ rmendivel@undc.edu.pe \\ Código Orcid: https://orcid.org/0000-0002-3147-2655 \\ MirIAM ANGOMA ASTUCURI ${ }^{* * *}$ \\ Universidad Nacional de Cañete, Lima-Perú \\ mangoma@undc.edu.pe \\ Código Orcid: https://orcid.org/0000-0002-4436-1276
}

Forma sugerida de citar: Oseda, Dulio, Mendivel, Ruth, \& Angoma, Miriam (2020). Estrategias didácticas para el desarrollo de competencias y pensamiento complejo en estudiantes universitarios. Sophia, colección de Filosofía de la Educación, 29, pp. 235-259.

* Licenciado en Educación, e Ingeniero de Sistemas y Computación. Magíster en Educación. Doctor en Ciencias de la Educación. Doctor en Psicología Educacional y Tutorial. Doctor en Sistemas de Ingeniería y PhD. in Business Administration USA. Es Profesor Principal de la Universidad Nacional de Cañete del Perú, Director de la Escuela Profesional de Ing. de Sistemas, conferencista internacional de la OIICE e investigador Renacyt-Concytec.

** Licenciada en Educación. Magíster en Administración de la Educación. Doctora en Educación. Es Docente A1 de la Universidad Nacional de Cañete del Perú, Conferencista Internacional de la OIICE.

*** Ingeniera de Sistemas y Computación. Magíster en Administración con mención en Gestión de Proyectos. Doctora en Sistemas de Ingeniería. Es Docente A1 de la Universidad Nacional de Cañete del Perú. 
Didactic strategies for the development of competences and complex thinking

\title{
Resumen
}

La presente investigación parte del marco lógico y teórico del desarrollo de competencias y el pensamiento complejo en el sistema universitario mundial, en ese sentido la investigación tuvo como objetivo demostrar los efectos de la aplicación de las estrategias didácticas para el desarrollo de competencias y pensamiento complejo en la carrera de Ingeniería de Sistemas en una universidad pública de Lima provincias. La investigación fue de tipo aplicada, nivel explicativo, se trabajó con un diseño pre experimental. La población la conformaron por 325 estudiantes de la carrera profesional de Ingeniería de Sistemas, y la muestra fue tomada no probabilísticamente por 23 estudiantes del X ciclo. El nivel de desarrollo de competencias fue porcentualmente del 74\% y del pensamiento complejo el $64,25 \%$. Se concluye en base a las tres estrategias didácticas utilizadas (estrategia basada en problemas, estrategias de aprendizaje colaborativo, y la estrategia incorporada de las tecnologías de información y comunicación) con un nivel de significancia del 5\% y un (p-valor: 0,006<0,050) que se ha desarrollado favorablemente las competencias y el pensamiento complejo en los estudiantes de la Carrera Profesional de Ingeniería de Sistemas en la Universidad Nacional de Cañete-Lima, Perú.

\section{Palabras clave}

Estrategias didácticas, desarrollo de competencias, pensamiento complejo, estrategia basada en problemas, estrategia colaborativa, incorporación de las TIC.

\begin{abstract}
The present investigation starts from the logical and theoretical framework of the development of competences and complex thinking in the world university system. In this sense, the research aimed to demonstrate the effects of the application of didactic strategies for the development of competences and complex thinking in the Systems Engineering degree at a public university in Lima provinces. The research was of an applied type, explanatory level, we worked with a preexperimental design. The population was made up of 325 students from the professional career of Systems Engineering, and the sample was taken non-probabilistically by 23 students of the X cycle. The level of development of competencies was $74 \%$ in percentage terms and $64,25 \%$ in complex thinking. It is concluded based on the three didactic strategies used (problem-based strategy, collaborative learning strategies, and the incorporated strategy of information and communication technologies) with a significance level of $5 \%$ and a (p-value: $0,006<0,050)$ that competencies and complex thinking have been favorably developed in the students of the Professional Career in Systems Engineering at the National University of Cañete, Lima, Peru."
\end{abstract}

\section{Keywords}

Didactic strategies, development of competences, complex thinking, problem-based strategy, collaborative strategy, incorporation of ICT.

\section{Introducción}

La excesiva y masiva expansión de la educación superior universitaria en el mundo y específicamente en el Perú según precisan Brooks, Waters y Pimlott-Wilson (2012); Yeom (2016) han hecho que la oferta de graduados en todas las carreras profesionales cada vez sea más amplia y diversificada, y por ende exista mayor competencia a la hora de postular a un trabajo. 
Esto definitivamente tiene impacto en la empleabilidad, según Figuereido, Biscaia, Rocha y Teixeira (2017) se ha evidenciado en ciertos desajustes en las habilidades, destrezas y competencias requeridas por el mercado laboral, así como también por aquellas formadas en la universidad.

En esta perspectiva como señalan Guzzomi, Male y Miller (2015); y Medland (2016) para convertirse en un buen profesional no solo es necesario tener el dominio del componente cognitivo y las habilidades técnicas/operativas de la especialidad, sino también ahora se exige el desarrollo de las habilidades sociales, habilidades blandas y las habilidades de pensamiento complejo necesarias en el lugar de trabajo.

En el mercado ocupacional, para Kalyuga, Renkl y Pass (2010); y Oliveri y Markle (2017) cada vez es más selectivo y restringido algunas de las competencias más requeridas por los empleadores son la capacidad de resolver problemas, el pensar críticamente y el comunicarse adecuadamente. Morales y Zambrano (2016) sostienen que, si bien estas habilidades forman parte de los perfiles de egreso de la mayoría de las universidades declarados, estos no cuentan con un adecuado seguimiento de las trayectorias y logros debidamente sistematizados en un sistema de información.

Asimismo, hoy en día merced a las políticas de acreditación y licenciamiento de las universidades y carreras profesionales en el país, en gran parte de las universidades tanto públicas como privadas, los programas de pregrado se han movido hacia un modelo basado en competencias precisan Knight (2011); Manzanares y Santamaría (2016). Aun cuando sus ventajas están relacionadas con procesos educativos más dinámicos y prácticos, para Pavié (2011); y Tobón (2013) estos buscan la formación integral de los estudiantes y la evaluación de los aprendizajes al momento de su egreso, se ha encontrado que entre las principales dificultades de su implementación está la escasa modificación que han tenido los procesos evaluativos.

Por un lado, según precisan Ampuero y Casas (2013) no hay coherencia entre las instituciones de educación superior universitaria en torno a lo que involucra la evaluación de competencias y, por otro lado, según indican Möller y Gómez (2014), se carece de instrumentos de evaluación coherentes y pertinentes para medir el pensamiento complejo.

En esa óptica se formula el problema de investigación:

¿Cuáles son los efectos de la aplicación de las estrategias didácticas en el desarrollo de las competencias y el pensamiento complejo en los estudiantes de la Carrera de Ingeniería de Sistemas en la Universidad Nacional de Cañete?

La investigación se justifica, en la medida que no posibilitó conocer su justificación en los siguientes componentes: 
- Conveniencia, este aspecto es fundamental, ya que abordar la temática de estudio de la investigación es conveniente para este tiempo y espacio, pues sirvió para conocer los niveles de logro de las competencias y el pensamiento complejo en la muestra de estudio.

- Relevancia social, la investigación se justifica en la medida que los directivos, docentes y estudiantes mejoren su conciencia social y moral, y esto sirva como punto de partida para la posterior acreditación de la carrera profesional, le cual redundará en una mejor sociedad.

- Implicaciones prácticas, como se midió el desarrollo de competencias y pensamiento complejo de los estudiantes universitarios en dos momentos, antes y después de la aplicación de las estrategias didácticas; esta aplicación ha sido favorable para los estudiantes de la carrera profesional de Ingeniería de Sistemas.

- Valor teórico, la información recopilada y procesada dentro del sistema universitario, servirá de sustento teórico para esta y otras investigaciones a fines, ya que enriquecerá el marco teórico y/o cuerpo de conocimientos que existe sobre el tema en mención.

- Utilidad metodológica, porque se siguió todas las etapas y/o fases del método científico en su consecución.

Respecto a la revisión de literatura, se tiene los aportes de Villarroel y Bruna (2019) quienes proponen una evaluación auténtica en la educación superior universitaria. Los instrumentos de evaluación de los aprendizajes, que tradicionalmente se utilizan en la universidad, presentan grandes debilidades a la hora de medir el conocimiento profundo de los estudiantes. En cambio, la evaluación auténtica otorga pertinencia al vincular lo que ocurre en las aulas con la vida real y laboral, midiéndose saberes en contextos reales.

Asimismo, Tapia y Luna (2010) presentaron la investigación "Validación de una prueba de habilidades de pensamiento para estudiantes de cuarto y quinto de secundaria y primer año de universidad", donde concluyen que el proceso de validación de la prueba comprendió un análisis de la validez de contenido a través de la evaluación por jueces y la evaluación psicométrica realizada en dos fases, la primera con una muestra piloto de validación para determinar la confiabilidad de la prueba a través del análisis de dificultad de los ítems, la correlación ítem-test y la estimación del coeficiente de Cronbach. Como resultado de este análisis fueron invalidados trece ítems, de los cuales se eliminaron definitivamente cuatro. La segunda fase consistió en reevaluar los nueves ítems invalidados, 
previa una revisión y mejora, en una muestra del grupo de estudiantes para la normalización. Un aspecto fundamental de la evaluación psicométrica lo constituyó el Análisis Factorial que permitió fundamentar la teoría en que se basa el instrumento. Se identificaron tres factores subyacentes: inferencia lógica deductiva e inductiva, clasificación, generalización y razonamiento analógico.

Barberousse (2007) en su investigación "Fundamentos teóricos del pensamiento complejo de Edgar Morin", donde concluye que ante lo que consideró la crisis del paradigma occidental de simplificación y disyunción, basado en la reducción y separación de los saberes, Morin planteó la emergencia de un nuevo paradigma de la complejidad, el cual intentaría articular y contextualizar las culturas científicas, las culturas de las humanidades y las culturas artísticas. Para llevar adelante tal propósito, se basó en la integración de ideas, conceptos y nociones provenientes de diversas fuentes teóricas.

Y Gonzáles (2002) en su investigación "El bucle educativo: Aprendizaje, pensamiento complejo y transdisciplinariedad", donde concluye que el aprendizaje como sistema complejo denota varias aristas y formas de presentarse en la metacomplejidad educativa. Un estudiante, antes de aprender como un momento único en su vida, debe afrontar, como una espiral intersubjetiva donde el desaprendizaje y reaprendizaje son dos componentes del aprendizaje de manera individual y social. El aprendizaje es la complejización de lo que el sujeto quiere aprender.

En las bases teórico-científicas, se va a analizar en primer lugar a la variable independiente referido a las estrategias didácticas. Según Ordóñez, et al. (2011) la estructuración propuesta en los diferentes trabajos busca modificar el proceso que regularmente es realizado en un aula de clase para lograr el desarrollo de competencias y pensamiento complejo de los estudiantes. La reformulación de las estrategias docentes bajo el enfoque del desarrollo de competencias y el pensamiento complejo consiste en revisar aquellas que ya hemos utilizado como son: el aprendizaje basado en problemas, el aprendizaje colaborativo y la incorporación de las TIC en el proceso de aprendizaje de los estudiantes universitarios.

Lo más pertinente es que, una vez que se han definido las competencias y aprendizajes complejos a lograr, es necesario diseñar el proceso de enseñanza-aprendizaje para su desarrollo y logro. La investigación educativa ha propuesto que el aprendizaje complejo y las competencias se logran cuando los estudiantes se enfrentan a situaciones que requieren la aplicación de estas.

Diversos autores han propuesto y demostrado la importancia de trabajar con problemas, casos, proyectos y tareas integradoras. En base a 
la revisión de literatura especializada, se tiene el sustento de tres estrategias didácticas a trabajar, considerada como dimensiones de la variable independiente:

- La estrategia del aprendizaje basado en problemas (ABP).

- La estrategia de aprendizaje colaborativo (EAC).

- La incorporación de las Tecnologías de Información y Comunicación (TIC).

Se comenzará analizando la primera estrategia, que fue iniciada en la Universidad de McMaster Ontario, Canadá, que introduce el aprendizaje basado en problemas (PBL por sus siglas en inglés, o ABP por sus siglas en español) en 1969 en la escuela de Medicina. Su propósito era mejorar la calidad de la educación médica, para transformar el currículum de un conjunto de temas hacia una organización alrededor de problemas de la vida real que requiere la integración de diferentes áreas del conocimiento para solucionar los problemas.

Para Ordóñez et al. (2011) la estrategia basada en problemas es un enfoque instruccional y curricular centrado en el estudiante que favorece que ellos investiguen, integren la teoría con la práctica, asimismo apliquen sus conocimientos, habilidades y actitudes para desarrollar una solución para un problema específico. Sin embargo, esta propuesta se ha complementado con las aportaciones que han hecho diferentes investigadores sobre diseño instruccional, como son Gagné que muestra la importancia de motivar a los estudiantes, informar los objetivos de aprendizaje, recuperar los aprendizajes previos, presentar los contenidos, proporcionar guías de aprendizaje, practicar la aplicación o ejecución, proporcionar retroalimentación, evaluar el desempeño y promover la retención y transferencia; también se tiene a Merrill que enfatiza que el aprendizaje se logra con la resolución de problemas de la vida real, cuando los aprendizajes previos se activan para generar nuevo conocimiento y aprendizaje, cuando se le demuestran al estudiante nuevos aprendizajes, cuando el estudiante puede aplicar el nuevo conocimiento y aprendizaje, y puede integrar los conocimientos y aprendizajes nuevos en su mundo real.

La estrategia de aprendizaje basado en problemas para Murray (1995) es una de las estrategias más utilizadas para integrar conocimientos, habilidades y actitudes y desarrollar y transferir habilidades para la resolución de problemas. Una de las contribuciones más importante para esta estrategia es la heurística de McMaster para resolver problemas que se resume en seis pasos: comprometerse, definir, explorar, planear, hacerlo y evaluar. 
La propuesta del aprendizaje basado en problemas además enfatiza el desarrollo del aprendizaje de habilidades de grupo, la proposición de alternativas y presentaciones. Los estudiantes se enfrentan a la búsqueda de alternativas de solución y del sustento teórico para ello.

El aprendizaje basado en problemas según Ordóñez et al. (2011) permite que los estudiantes descubran por sí mismos las relaciones de la teoría con la situación problemática propuesta y el profesor coordina, aclara y enfatiza los aspectos importantes de estas relaciones. El reto de resolver un problema y las dificultades que van sorteando fortalece el aprendizaje, la participación y el liderazgo de los equipos.

El diseño de una asignatura con la estrategia didáctica basado en problemas permitirá cubrir todos los temas teóricos a través de su aplicación práctica. El aprendizaje basado en problemas, según Ordóñez et al. (2011) incluye varias fases:

- Planteamiento del problema. "El problema es planteado por el profesor y discutido con los estudiantes. Se da un plazo de para concluir dicho trabajo."

- Apropiación del problema. El problema es abierto, puede ser resuelto mediante diferentes abordajes y los estudiantes deben hacer suposiciones, selección de parámetros que deben justificar. La aplicación de criterios independientes lleva a soluciones diferentes."

- Programación del trabajo. "Los estudiantes se pueden organizar en grupos para investigar los aspectos teóricos que sustentan la solución del problema y resolverlo con las herramientas y técnicas apropiadas para el problema."

- Solución del problema. "La solución incluye la evaluación y discusión sobre los resultados obtenidos, que se comparan con criterios previamente determinados y comunicados a los estudiantes."

- Elaboración de "un informe, que puede ser presentado de forma individual o en grupo. Generalmente se incluyen las siguientes secciones: introducción, objetivos generales y específicos, planteamiento del problema y metodología de solución, resultados obtenidos y su discusión, conclusiones y recomendaciones, bibliografía."

- Presentación de "resultados al grupo. El propósito es que los estudiantes potencien la competencia del trabajo en grupo, de forma que puedan aclarar sus dudas en su propio lenguaje, que ellos mismos sean sus propias guías en el grupo y que interioricen la materia estudiada. También se propicia la competencia 
de exponer en público los resultados, defenderlos y discutirlos y presentarlos en forma escrita tal y como se acostumbra en un informe de investigación."

Con este tipo de estrategia según Ordóñez et al. (2011) se promueve:

- El "aprendizaje de conocimientos en los temas abordados en el curso, su integración con la temática general y su aplicación a casos particulares de problemas en las carreras de ingeniería y profesiones a fines."

- El desarrollo de la "competencia de trabajo en grupo y la presentación escrita de los resultados de la investigación tanto básica como aplicada."

- El desarrollo de la competencia "de exposición oral, la defensa de resultados y el diálogo en público.

- El aprendizaje basado en problemas bajo el enfoque de la complejidad puede reformularse considerando las implicaciones de las soluciones en los contextos sociales, económicos, ambientales y éticos. Se incluyen, en la defensa de los resultados, los argumentos y los criterios para seleccionar la mejor alternativa."

Ahora se analiza la segunda propuesta, la estrategia de aprendizaje colaborativo EAC), según Ordóñez et al. (2011) la EAC favorece el desarrollo de las habilidades, no sólo en el campo específico de la asignatura, sino también en el campo ético (responsabilidad y solidaridad), comunicativo (debates, sustentación y argumentación), emocional (interdependencia positiva, interacción conducente a resultados, apoyo, ayuda mutua, superación de debilidades, logro de resultados, etc.) y actitudinal (compartir conocimientos, mejoramiento continuo, autoevaluación permanente, etc.).

Para que una metodología adquiera la connotación de colaborativa es esencial que se cumplan cinco principios básicos según Ordónez et al. (2011):

- El "primer principio, es la interdependencia positiva, considerada como la estrategia en la que los estudiantes asimilen que están entrelazados con otros en el sentido en que, si algún miembro del grupo sale perjudicado, el grupo sale perjudicado y si cada miembro del grupo supera un objetivo es una superación de todo el grupo, esto es, o todos ganan o todos pierden".

- El "segundo principio, es que se promueve la interacción cara a cara entre estudiantes. Esta interacción cara a cara se da cuando 
los estudiantes entre sí se ayudan, se asisten, se motivan y se colaboran en los esfuerzos de cada uno por aprender. Los estudiantes pueden promover el aprendizaje de cada uno de los demás por medio de explicación oral acerca de cómo resolver problemas, discutiendo entre sí la naturaleza de los conceptos y estrategias aprendidas, compartiendo sus conocimientos y explicando las conexiones entre el aprendizaje pasado y el presente".

- El "tercer principio, es la responsabilidad individual, ejercida cuando se evalúa el desempeño de cada miembro del grupo y los resultados obtenidos permiten realimentar al grupo y al mismo individuo. Es fundamental que los miembros del grupo conozcan quienes requieren mayor asistencia para completar la labor asignada y que no puedan perjudicar el trabajo de los otros. Un método común de estructurar la responsabilidad individual consiste en asignar tareas al grupo o una tarea individual a cada estudiante y seleccionar aleatoriamente a un estudiante para que represente los esfuerzos de todo el grupo".

- El "cuarto principio es la formación social, los grupos no pueden funcionar efectivamente si los estudiantes no tienen o ejercen el liderazgo, la toma de decisiones, construcción de verdades, la comunicación y el manejo de conflictos. Estos elementos de formación deben tomarse completamente como propósitos académicos".

- El "quinto principio, lo constituye el proceso grupal, determinado por el aseguramiento de que los grupos trabajen como tal alcanzando sus metas y manteniendo una relación efectiva de trabajo entre sus miembros".

Y la tercera propuesta didáctica fue la incorporación de las Tecnologías de Información y Comunicación (TIC) en los procesos de enseñanza y de aprendizaje, para el mismo Ordóñez et al. (2011) con la incursión de las TIC en los programas de formación se ha planteado la transformación de una formación basada en contenidos hacia conceptos de amplio espectro y de fortalecimiento de principios básicos, lo cual requiere de nuevas estrategias pedagógicas para el proceso de aprendizaje.

Las TIC aportan a la implementación de una educación de tercera generación apoyada en el uso de nuevos métodos, técnicas, estrategias y medios para una formación integral. Las TIC ofrecen herramientas y recursos a través de objetos de aprendizaje, que constituyen un entorno propicio para el aprendizaje colaborativo y potencian el desarrollo de actitudes de autoformación y habilidades de búsqueda, selección, valo- 
ración y organización de la información. Las instituciones de formación universitaria están utilizando estas tecnologías como recurso didáctico para el desarrollo de los contenidos de cada asignatura, y como herramienta para flexibilizar los entornos de enseñanza aprendizaje.

En las actividades pedagógicas, según Ordóñez et al. (2011) las TIC ofrecen un amplio espectro de recursos, que buscan facilitar el aprendizaje significativo y personalizado de conceptos complejos, así como la construcción y confrontación de conocimientos, en ambientes interactivos y dinámicos, altamente llamativos. Según Trigos (2001) existen tres criterios para tener en cuenta para lograr procesos de aprendizaje en la red:

- Que se "realice en red, lo que permite actualización, almacenaje, recuperación y distribución inmediata de contenidos y de información".

- Que se "haga llegar al dicente a través de un computador utilizando estándares tecnológicos de Internet".

- Que "esté centrado en la más amplia visión de soluciones de aprendizaje que vayan más allá de los paradigmas tradicionales de la formación".

La incorporación de las TIC a los procesos de enseñanza y de aprendizaje permiten entre otras las siguientes actividades:

- Establecimiento "de una vía de comunicación permanente con los estudiantes a través de una plataforma virtual".

- Desarrollo "de materiales de soporte de las asignaturas por parte de los docentes y su ubicación en una plataforma".

- Revisión de "herramientas de soporte para el proceso de aprendizaje desarrolladas en otras universidades para su utilización en línea por parte del estudiante".

- Desarrollo de "objetos de aprendizaje para entornos virtuales que le permitan a los estudiantes realizar actividades de aprendizaje en línea sin la presencia de los docentes".

- Diseño "de sistemas de autoevaluación y evaluación en línea".

- Diseño "de materiales y herramientas para el estudio independiente de estudiantes con diferentes niveles de aprendizaje".

- Disposición "de alternativas para un aprendizaje flexible en tiempo y ruta”.

Ahora se analizará la variable dependiente denominado desarrollo de competencias y el pensamiento complejo. 
El concepto de competencia es tan propio del mundo laboral, que se ha incorporado en el horizonte universitario como un catalizador de modelos curriculares desde la década del 90 asumiendo distintas denominaciones: formación por competencias, planes de estudio basados en el enfoque por competencias, propuestas educativas por competencias; todo con miras a transformarse en una opción de formación académica.

Hay bastante consenso en justificar esta decisión, lo que puede resumirse principalmente en la característica principal de la época que nos ha correspondido vivir. Para Manríquez (2012) una época de cambios exponenciales de todo orden y magnitud, lo que está provocando un desplazamiento del centro de gravedad de la enseñanza universitaria, históricamente encargada de formar profesionales para el mercado laboral, cuya estructura cada vez más se orienta hacia el nivel terciario. Consecuentemente, el contenido de variadas ocupaciones está cambiando rápidamente, generándose nuevas demandas de competencias y conocimientos.

Todo ello en el contexto de la globalización y las nuevas tecnologías de la información que evolucionan incesantemente, según precisa Brunner (2003).

Uno de los problemas que plantea la evaluación de competencias es que ellas necesariamente son el producto de un proceso secuenciado; la evaluación debería propender a la constatación del dominio de una competencia, lo que difícilmente puede ser determinada a través de un solo método. Para fijar ideas, nos referiremos a la llamada Pirámide de Miller, un modelo para evaluar competencias planteado desde la década de los 90 por Miller (1990) en el ámbito de la enseñanza universitaria y que presentamos en la Figura 1:

Figura 1

Pirámide de Miller (1990)

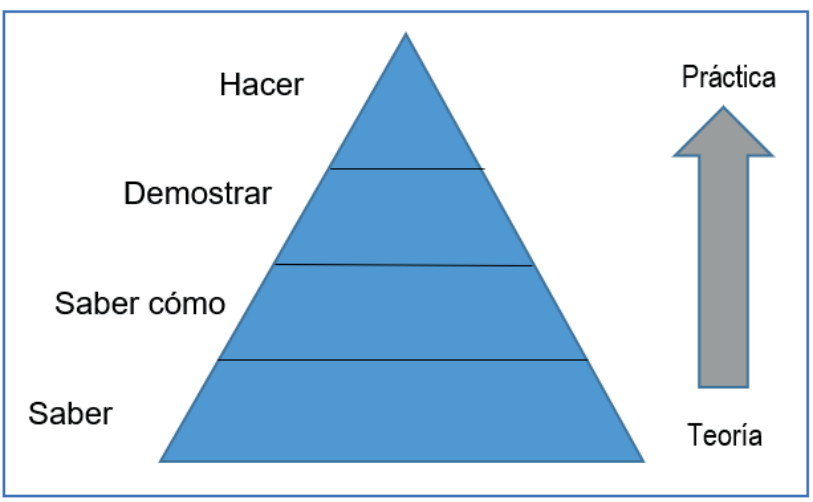


La figura muestra con claridad las etapas que debe escalarse para considerar una competencia desarrollada. Las dos primeras etapas (base) están evidentemente relacionadas con lo cognitivo y los dos superiores con el comportamiento. Es relativamente obvio que los niveles denominados saber y saber cómo, podrían evaluarse a través de instrumentos tradicionales en el contexto de un modelo que intenta certificar el dominio de tópicos tratados por el docente en clases, con énfasis en pruebas de papel y lápiz y que de alguna manera refuerzan el pensamiento convergente.

Para Manríquez (2012) en un enfoque de competencias, esta práctica vendría corresponder al ámbito de lo que se define como capacidad - componente específico que forma parte de una competencia- definida en términos de acciones sobre contenidos ejecutados en torno a tareas que cobran sentido siempre y cuando estén dentro de un contexto de la propia capacidad que se pretenden evidenciar, lo que presenta la restricción de que los constructos en este escenario miden conocimientos genéricos, es decir, apuntarían hacia competencias generales y no necesariamente en un contexto adecuado. Esto último no es menor: supongamos que se está evaluando la tarea medir una altura; esta tarea debería estar en el contexto de una acción que estuviera conectada con una competencia profesional, más allá de la aplicación experimental de método científico en general.

Respecto al pensamiento complejo Peña (2018) considera que, la teoría de la complejidad y el pensamiento complejo intenta articular dominios disciplinarios a favor del docente del futuro, quebrados por el pensamiento disgregador y aspira al conocimiento multidimensional.

De allí que Morín (2003), indica que el pensamiento complejo "es la capacidad de interconectar distintas dimensiones de lo real. Promueve un enfoque transdisciplinario y holístico, sin abandonar la noción de las partes constituyentes del todo" (p. 5).

La teoría de la complejidad capta la realidad como sistema complejo, en sus diversas conexiones, mediaciones y condicionamientos. Por eso no establece relaciones antitéticas entre orden y caos, incertidumbre y certidumbre, entre las partes y el todo. Si no lo asume teniendo conciencia de que son antitéticas, cada uno por separado, pero a la vez, los unifica, sin convertirlos en un todo, cada elemento conserva su identidad y unidad.

Ahora bien, la formación universitaria hacia la transformación requiere aprobación especializada, posiblemente de carácter pedagógico, es necesario considerar que las políticas institucionales, el aseguramiento de la calidad que potencie aquellas que promuevan el desarrollo profesional en las universidades, a lo largo de su carrera, por ello, dado que en este momento las universidades deben desarrollar un escenario orientado a 
potenciar la formación pedagógica y el desarrollo profesional como una estrategia fundamental para mejorar la calidad de la enseñanza. El docente en su práctica cotidiana, integra distintos saberes desde esta perspectiva, el saber docente está formado por una mixtura más o menos coherente de saberes curriculares y experienciales.

Estas consideraciones según investigadores como García (2007), señala que "la práctica docente no sólo es un objeto del saber de las ciencias de la educación sino también una actividad que teoriza, diversos saberes que pueden llamarse pedagógicos, los cuales se presentan como doctrinas por la práctica educativa" (p. 57). Ampliando el término, las reflexiones personales y normativas conducen al sistema, más o menos coherente en los saberes pedagógicos en representación de la actividad educativa en el sistema universitario.

Teniendo como base, la revisión de literatura especializada, la investigación tuvo como objetivo demostrar los efectos de la aplicación de las estrategias didácticas para el desarrollo de competencias y pensamiento complejo en los estudiantes de la Carrera de Ingeniería de Sistemas en una universidad pública de Lima provincias. Y la hipótesis la aplicación de las estrategias didácticas produce efectos significativos en el desarrollo de competencias y el pensamiento complejo en los estudiantes de la Carrera de Ingeniería de Sistemas en la Universidad Nacional de Cañete.

\section{Material y métodos}

La investigación fue del tipo aplicada y nivel explicativo. Como método de investigación general utilizó el método científico y como métodos específicos, el experimental, el estadístico y el hipotético deductivo.

Al respecto, Ávila (2001) afirma que "la investigación aplicada está interesada en la aplicación de los conocimientos a la solución de un problema práctico inmediato, busca conocer para hacer, para actuar, para construir, para modificar, le preocupa la aplicación inmediata sobre una realidad concreta" (p. 38).

Según Pardinas (2004) “el método científico consiste en la sucesión de pasos que debemos dar para descubrir nuevos conocimientos o, en otras palabras, para comprobar hipótesis que explican o predicen conductas de fenómenos desconocidos hasta el momento" (p.72).

La población del estudio estuvo conformada por 325 estudiantes debidamente matriculados en el ciclo 2019-II, todos ellos pertenecientes 
a la Carrera Profesional de Ingeniería de Sistemas de la Universidad Nacional de Cañete.

La muestra de estudio fue tomada no probabilísticamente y la conformaron 23 estudiantes del X ciclo de la universidad.

Luego de haber seleccionado el diseño de investigación apropiado que fue el pre-experimental de acuerdo con el problema de estudio y objetivos, el esquema se muestra a continuación:

GE: $0, X_{1} 0_{2}$

Donde:

GE: grupo experimental único.

$0_{1}$ : Aplicación del Pre test.

$0_{2}$ : Aplicación del Post test.

X: Manipulación de la variable independiente.

Se ha diseñado varios instrumentos de investigación que fueron las pruebas pedagógicas tipo objetivas/ensayo para medir el componente cognitivo de las competencias, una rúbrica para medir el componente procedimental y una escala de actitudes para medir el componente actitudinal, así como también el inventario para medir del pensamiento complejo, los mismos que pasaron previamente por los criterios de Confiabilidad (Alfa de Cronbach $=0,964$ ) y Validación de Constructo (Análisis de factores confirmatorio $=0,992$ ) luego se procedió a la recolección de datos, para ello primero se procedió con las cartas de consentimiento informado a los integrantes de la muestra de estudio especificada para proceder con la ejecución práctica de la investigación Pablo (2007).

\section{Análisis y resultados}

Antes de aplicar las tres estrategias didácticas en mención (estrategia basada en problemas, estrategias de aprendizaje colaborativo, y la estrategia incorporada de las tecnologías de información y comunicación), se tuvo los siguientes resultados: 
Figura 1

Desarrollo de competencias Pre-test

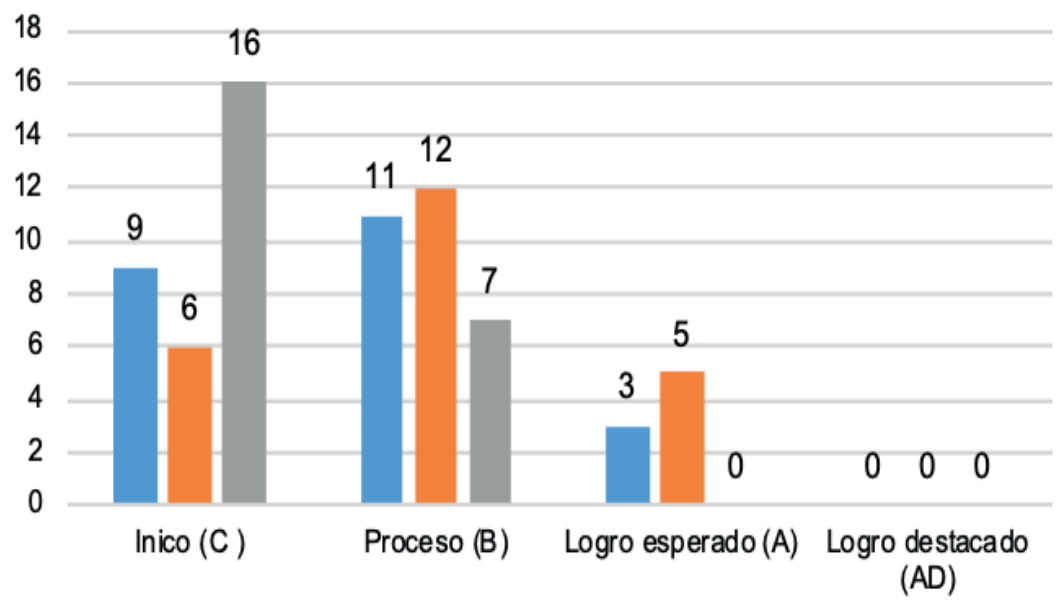

(AD)

=Componente cognitivo $=$ Componente procedimental $=$ Componente actitudinal

Fuente: Base de datos de los investigadores.

Respecto al logro de competencias se tiene que, en el pre test, de los 23 estudiantes de la carrera de Ingeniería de Sistemas, en el componente cognitivo, se tiene a 9 estudiantes en el nivel inicio (C), 11 en el nivel en proceso (B), y 3 en el nivel logro esperado (A), en el nivel logro destacado (AD) no se tuvo a ningún estudiante. En el componente procedimental, se tiene a 6 estudiantes en el nivel inicio (C), 12 en el nivel en proceso (B), y 5 en el nivel logro esperado (A), y en el nivel logro destacado (AD) tampoco se tuvo a ningún estudiante. Y finalmente en el componente actitudinal es todavía más bajo las puntuaciones, ya que se tuvo a 16 estudiantes en el nivel inicio (C), 7 en el nivel en proceso (B), y ningún estudiante en el nivel logro esperado $(A)$ y logro destacado $(A D)$ respectivamente el cual fue muy preocupante.

Ahora, se apreciará los resultados en la dimensión pensamiento complejo. 
Figura 2

Desarrollo del pensamiento complejo Pre-test

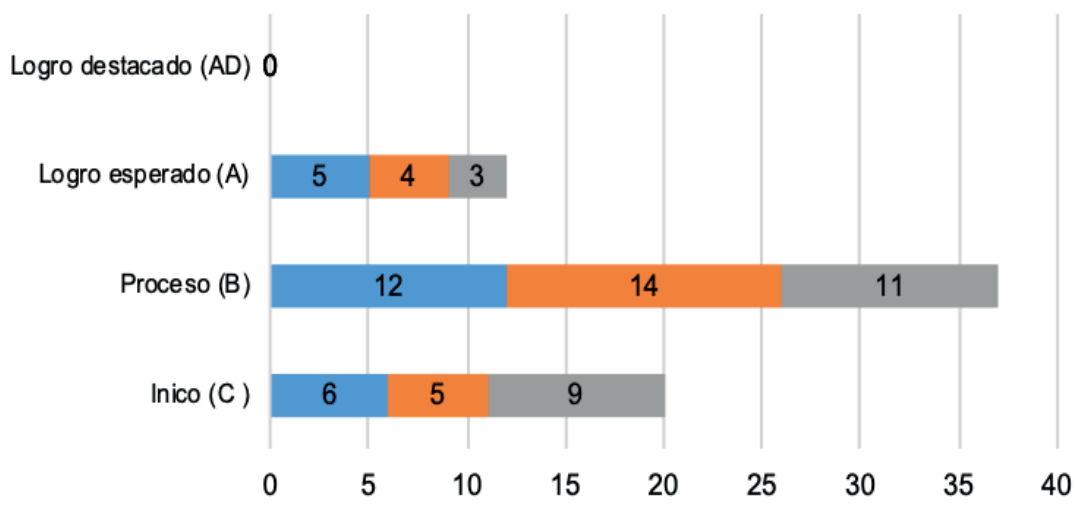

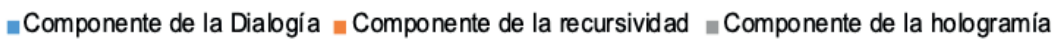

Fuente: Base de datos de los investigadores.

Respecto a los niveles de logro del pensamiento complejo también en el pre test antes de la aplicación de las tres estrategias didácticas se tuvo que, de los 23 estudiantes de la carrera de Ingeniería de Sistemas, en el componente dialogía, se tuvo a 6 estudiantes en el nivel inicio (C), 12 en el nivel en proceso (B), y 5 en el nivel logro esperado (A), en el nivel logro destacado $(\mathrm{AD})$ no se tuvo a ningún estudiante. En el componente recursividad, se tuvo a 5 estudiantes en el nivel inicio (C), 14 en el nivel en proceso (B), y 4 en el nivel logro esperado (A), y en el nivel logro destacado $(\mathrm{AD})$ tampoco se tuvo a ningún estudiante. Y finalmente en el componente hologramía se tuvo a 9 estudiantes en el nivel inicio (C), 11 en el nivel en proceso (B), y en el nivel logro esperado a 3 estudiante, y en el nivel logro destacado (AD) no se tuvo a ningún estudiante.

Veamos ahora los resultados del post test, posterior a la aplicación de las tres estrategias didácticas (estrategia basada en problemas, estrategias de aprendizaje colaborativo, y la estrategia de incorporación de las tecnologías de información y comunicación), planificado en 15 sesiones de aprendizaje durante el ciclo 2019-II, cuyos resultados se muestran a continuación: 
Figura 3

Desarrollo de competencias Post-test

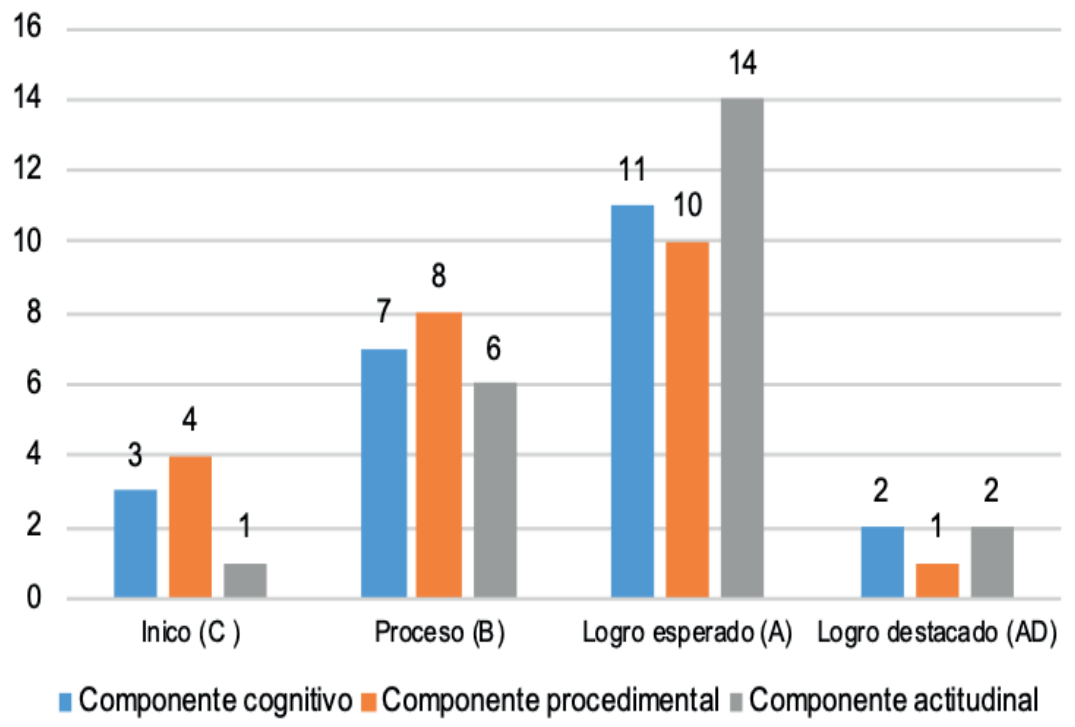

Fuente: Base de datos de los investigadores.

Respecto al logro del desarrollo de las competencias se tuvo en el post test, de los 23 estudiantes de la carrera de Ingeniería de Sistemas, en el componente cognitivo, se tiene a 3 estudiantes en el nivel inicio (C), 7 en el nivel en proceso (B), y 11 en el nivel logro esperado (A), y a 2 estudiantes en el nivel logro destacado (AD) siendo esto un buen indicador de logro. En el componente procedimental, se tuvo a 4 estudiantes en el nivel inicio (C), 8 en el nivel en proceso (B), 10 en el nivel logro esperado (A), y en el nivel logro destacado (AD) a un solo estudiante. Y finalmente en el componente actitudinal es ahora todavía mejor, ya que se tuvo a 1 estudiantes en el nivel inicio (C), 6 en el nivel en proceso (B), 14 estudiantes en el nivel logro esperado (A) y 2 estudiantes en el nivel logro destacado (AD). 
Figura 4

Desarrollo del pensamiento complejo Post-test

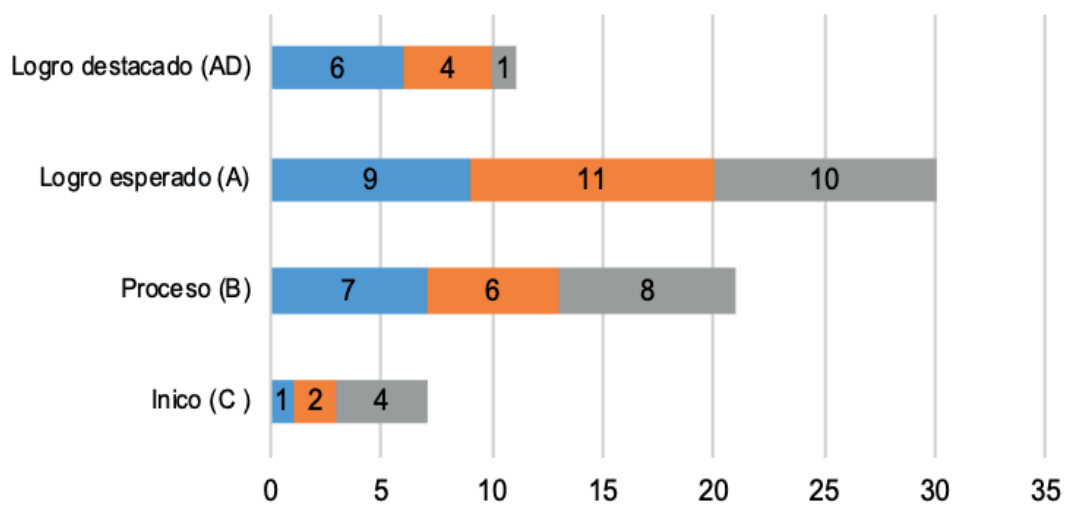

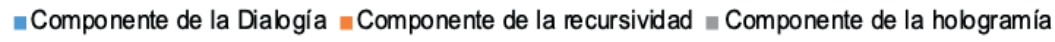

Fuente: Base de datos de los investigadores.

Respecto a los niveles de logro del pensamiento complejo también en el post test después de la aplicación de las tres estrategias didácticas se tuvo que, de los 23 estudiantes de la carrera de Ingeniería de Sistemas, en el componente dialogía, se tuvo a 1 estudiantes en el nivel inicio (C), 7 en el nivel en proceso (B), 9 en el nivel logro esperado (A), y en el nivel logro destacado (AD) se tuvo a 6 estudiantes el cual es muy favorable. En el componente recursividad, se tuvo a 2 estudiantes en el nivel inicio (C), 6 en el nivel en proceso (B), 11 en el nivel logro esperado (A), y en el nivel logro destacado (AD) se tuvo a 4 estudiantes. Y finalmente en el componente hologramía se tuvo a 4 estudiantes en el nivel inicio (C), 8 en el nivel en proceso (B), en el nivel logro esperado a 10 estudiante, y en el nivel logro destacado $(\mathrm{AD})$ a un solo estudiante.

Ahora bien, respecto a la prueba de hipótesis general, se utilizó el estadígrafo de Wilcoxon.

Tabla 1

Mann-Whitney Test. Ranks

\begin{tabular}{|l|l|l|l|l|}
\hline & \multicolumn{1}{|c|}{ Grupo } & \multicolumn{1}{c|}{ N } & \multicolumn{1}{c|}{ Mean Rank } & \multicolumn{1}{c|}{ Sum of Ranks } \\
\hline \multirow{4}{*}{ Evaluación } & Pre test & 23 & 90.64 & 16315.50 \\
\cline { 2 - 6 } & Post test & 23 & 270.36 & 48664.50 \\
\cline { 2 - 6 } & Total & 46 & & \\
\hline
\end{tabular}


Test Statistics(a)

\begin{tabular}{|l|l|}
\hline & \multicolumn{1}{c|}{ Evaluación } \\
\hline Mann-Whitney U & 25.500 \\
\hline Wilcoxon W & 1315.500 \\
\hline Z & -16.476 \\
\hline Asymp. Sig. (2-tailed) & .006 \\
\hline
\end{tabular}

a Grouping Variable: Grupo

La contrastación de hipótesis general.

Hipótesis nula: $\mathrm{H}_{\mathrm{o}}$ : La aplicación de las estrategias didácticas no produce efectos significativos en el desarrollo de competencias y el pensamiento complejo en los estudiantes de la Carrera de Ingeniería de Sistemas en la Universidad Nacional de Cañete.

Hipótesis alterna: $\mathrm{H}_{1}$ : La aplicación de las estrategias didácticas produce efectos significativos en el desarrollo de competencias y el pensamiento complejo en los estudiantes de la Carrera de Ingeniería de Sistemas en la Universidad Nacional de Cañete.

Nivel de significancia o riesgo: $\alpha=0,05$ o $5 \%$

Cálculo del estadístico de prueba: $\mathrm{N}=23$, Wilcoxon $\mathrm{W}=1350$

Decisión estadística: Puesto que (p-valor: 0,006<0,050), en consecuencia, se rechaza la hipótesis nula (Ho) y se acepta la hipótesis alterna (Hi).

Conclusión estadística: Se concluye que, la aplicación de las estrategias didácticas ha producido efectos favorables y significativos en el desarrollo de competencias y el pensamiento complejo en los estudiantes de la Carrera de Ingeniería de Sistemas en la Universidad Nacional de Cañete.

\section{Discusión y conclusiones}

Como se puede apreciar en las tablas estadísticas, como variable independiente se ha utilizado tres estrategias didácticas (estrategia basada en problemas, estrategias de aprendizaje colaborativo, y la estrategia incorporada de las tecnologías de información y comunicación), con ello se ha podido evidenciar sus resultados en la variable dependiente que es el desarrollo de competencias y el pensamiento complejo, cuyos resultados son favorables comparando el pre test y post test respectivamente, en ese sentido la evaluación del aprendizaje para Villarroel y Bruna (2019) "es una de las prácticas docentes que mayor impacto ha mostrado tener sobre el aprendizaje de los estudiantes. La forma en que este es evaluado 
configura la calidad de los resultados, habilidades y competencias que alcanzarán los aprendices" (p. 501).

Así mismo, aprender tiene relación con hacer uso del conocimiento para algo. Ese algo puede ser la comprensión de un fenómeno social, la resolución de un problema disciplinar, o bien la evaluación de la calidad o efectividad de un producto.

Definitivamente, según Manzanares y Santamaría (2016) la forma en que uno evalúa a los estudiantes es crucial al momento de medir, emitir un juicio valorativo y a la toma de decisiones tal como le menciona.

En la experiencia vivida, para medir el desarrollo de competencia se tuvo que utilizar hasta tres instrumentos según precisa Kerlinger y Lee (2002) primero la prueba pedagógica para medir el componente cognitivo, luego se utilizó una rúbrica para el componente procedimental y, finalmente para el componente actitudinal se utilizó la escala de actitudes. Y para la sub variable pensamiento complejo se utilizó un inventario, todo ellos pasaron obligatoriamente por el criterio de confiabilidad y validez previa a su aplicación.

Ahora, como una hubo una mejora significativa, se establece que Barberousse (2007) "la producción teórica no es nunca un intento de logro acabado, sino más bien un proceso que, en su devenir mismo, marca un rumbo cognitivo en el que somos invitados a participar" (p. 9). Su obra por lo tanto debe en consecuencia, ser entendida no sólo en términos de su contenido, sino también de su proceso productor.

En esa óptica urge la necesidad de innovar el proceso de evaluación, pero con sustento teórico que demanda una actividad holística donde involucra a todos los actores educativos, que en nuestro caso es la Universidad Nacional de Cañete.

Ahora, si bien es cierto para Murray (1995) "al enseñar, el docente no debe desarrollar una intervención caracterizada por su unidireccionalidad en la que la única voz a escuchar es la del propio docente, sino que hay que dar lugar a la voz del alumno" (p.154). Esto no es otra cosa que la manifestación de su capacidad para pensar y construir significados, del mismo modo en el proceso de evaluación debe encontrar el alumno un lugar para expresar los significados desde su propia perspectiva.

Tomando en consideración este proceso meta complejo de la educación y la visión de aula-mente-social como un elemento central de una nueva visión didáctica de la educación, que rompe los modelos tradicionales de enseñanza y aprendizaje, incorporo un nuevo elemento, el bucle educativo, es decir, el elemento de ida y vuelta de cualquier proceso de aprendizaje y enseñanza. Hay procesos de cambio educativo variable que llevan al estudiante a aprender, desaprender y reaprender el conocimiento. 
Para Gonzales (2002) el aula-mente-social es en sí misma un bucle, que en meta complejidad educativa permite el planteamiento de modelos de planificaciones de aula meta complejas con formas de espiral, icónicas, circulares, doble icono, y otras más. Más allá del modelo simple o reduccionista de conducir una clase convencional.

En base a los datos estadísticos existe suficientes evidencias para afirmar que el uso de las estrategias didácticas, han sido favorables para promover el desarrollo de las competencias y también el pensamiento complejo en el aula, en ese sentido Trigos (2011) concluye que estas estrategias corresponden a la necesidad de transversalidad en el aprendizaje y la formación integral. Así, en la actualidad, la Universidad del Rosario cuenta con unos programas de competencias comunes en las áreas de lenguaje, lógico-matemática y ética, que son impartidos en los primeros semestres de pregrado a través del Núcleo de Formación Rosarista y Núcleo Básico.

Sin embargo, se espera que las asignaturas que los estudiantes cursan a lo largo de la carrera promuevan y fortalezcan estas competencias iniciales, con el fin de que el estudiante vaya ganando en complejidad y destreza con el paso del tiempo en los ciclos superiores. Algo similar aconteció al inicio, en la Carrera Profesional de Ingeniería de Sistemas de la Universidad Nacional de Cañete, donde las asignaturas del plan de estudios 2016 han promovido y fortalecido las competencias generales del perfil de la carrera, además han servido para que los estudiantes de los últimos ciclos hayan consolidado en su formación la complejidad y destreza respecto al perfil del egresado en los últimos ciclos de la carrera.

Para Ordoñez, et al. (2011):

Llevar al aula los avances de la investigación sobre el aprendizaje, las necesidades y retos de la vida actual y del campo profesional, los cuestionamientos sobre la complejidad de la realidad y el uso de las TIC son algunos de los elementos que se han considerado en este proyecto, además de las propuestas de nuevos enfoques para la evaluación. La contribución de los profesores es su reflexión sobre el impacto que la innovación en sus prácticas docentes ha tenido en el logro de aprendizajes significativos por parte de los dicentes y, al mismo tiempo, la explicitación del proceso para transferir su experiencia (p. 205).

Las estrategias basado en problemas, el aprendizaje cooperativo y el uso de las TIC en el aula universitaria, específicamente en la Carrera de Ingeniería de Sistemas ha repercutido favorablemente, ya que no solo se manifiesta en logros académicos, sino también en habilidades y destrezas propios de la profesión, asimismo sin dejar de lado el aspecto actitudinal, 
sumado a la formación holística de resolución de problemas, mediante la experimentación propiamente dicho.

\section{Conclusiones}

Se ha demostrado con un nivel de significancia del $5 \%$, que las estrategias didácticas han favorecido significativamente ( $p$-valor: $0,006<0,050)$ el desarrollo de las competencias (74\%) y el pensamiento complejo $(64,25 \%)$ en los estudiantes de la Carrera Profesional de Ingeniería de Sistemas de la Universidad Nacional de Cañete en Lima-Perú.

Se ha determinado que, la estrategia didáctica basada en problemas ha favorecido significativamente (p-valor: 0,008<0,050) el desarrollo de las competencias y el pensamiento complejo en los estudiantes de la Carrera Profesional de Ingeniería de Sistemas de la Universidad Nacional de Cañete en Lima-Perú.

Luego también se ha determinado que, la estrategia del aprendizaje colaborativo ha favorecido significativamente ( $\mathrm{p}$-valor: $0,000<0,010$ ) el desarrollo de las competencias y el pensamiento complejo en los estudiantes de la Carrera Profesional de Ingeniería de Sistemas de la Universidad Nacional de Cañete en Lima-Perú.

Y finalmente también se ha determinado que, la incorporación de las tecnologías de información y comunicación ha favorecido significativamente (p-valor: $0,000<0,010$ ) el desarrollo de las competencias y el pensamiento complejo de los estudiantes de la Carrera Profesional de Ingeniería de Sistemas de la Universidad Nacional de Cañete en Lima-Perú.

\section{Bibliografía}

AMPUERO, Nancy \& CASAS, Madeline

2013 Evaluación de aprendizajes en el contexto de innovaciones curriculares en el pregrado en universidades chilenas. Santiago de Chile: Centro Interuniversitario de Desarrollo, Cinda.

ÁVILA, Roberto Benito

2001 Metodología de la Investigación. Lima: Estudio R.A.

BARBEROUSSE, Paulette

2007 Fundamentos teóricos del pensamiento complejo de Edgar Morin. Revista Electrónica Educare, 12(2), 95-113.

BROOKS, Router, WATERS, James, \& PIMLOTT-WILSON, Hilton

2012 International education and the employability of UK students. British Educational Research Journal, 38(2), 281-298. 
BRUNNER, Jerome

2003 Educación e Internet: ¿La próxima revolución? Santiago: Fondo de Cultura Económica.

FIGUEREIDO, Higs, BISCAIA, Romer, ROCHA, Veronika, \& TEIXEIRA, Peter

2017 Should we start worrying? Mass higher education, skill demand and increasingly complex landscape of young graduates' employment. Studies in Higher, 42(8), 1401-1420. https://doi.org/10.1080/03075079.2015.1101754

GARCÍA, Joel

2007 Estructura Organizativa de la Educación en Venezuela. Tesis para optar al título de doctor. Maracaibo, Venezuela: Universidad Rafael Urdaneta.

GONZÁLES, Juan Miguel

2002 El bucle educativo: Aprendizaje, pensamiento complejo y transdisciplinariedad. Modelos de planificaciones de aula metacomplejas. Revista Integra Educativa, 2(2), 83-101.

GUZZOMI, Alen, MALE, Sartre, \& MILLER, Karem

2015 Students' responses to authentic assessment designed to develop commitment to performing at their best. European Journal of Engineering Education, 42(3), 1-22. https:// doi.org/10.1080/03043797.2015.1121465.

KALYUGA, Sendy, RENKL, Alberti, \& PAAS, Ferdinand

2010 Facilitating flexible problem solving: A cognitive load perspective. Educational Psychology Review, 22, 175- 186. https://doi.org/10.1007/s10648-0109132-9.

KERLINGER, Fred, \& LEE, Howard

2002 Investigación del comportamiento. México DF: McGraw Hill.

KNIGHT, Berenice

2011 Training in professional psychology in the US: An increased focus on competency attainment. Australian Psychologist, 46, 140-141. https://doi. org/10.1111/j1742-9544.2011.00026.x.

MANRÍQUEZ, Luis

2012 ¿Evaluación en Competencias? Estudios Pedagógicos XXXVIII, 1(1), 353-366, https://doi.org/10.4067/SO718-07072012000100022.

MANZANARES, Alberto, \& SANTAMARÍA, José Sabina

2016 La dimensión pedagógica de la evaluación por competencias y la promoción del desarrollo profesional en el estudiante universitario. Revista Iberoamericana de Evaluación Educativa, 5(1), 187-202.

MEDLAND, Emerine

2016 Assessment in higher education: Drivers, barriers and directions for change in the UK. Assessment \& Evaluation in Higher Education, 41(1), 81-96. https://doi.org/10.1080/02602938.2014.982072.

MILLER, Gentz

1990 The assessment of Clinical Skill. Compotence-Performance. Academic Medicine, 9(65), 63-67.

MÖLLER, Irene \& GÓMEZ, Hasser

2014 Coherencia entre perfiles de egreso e instrumentos de evaluación en carreras de educación básica en Chile. Calidad en la Educación, 41, 17-49. https:// doi.org/10.4067/s0718- 45652014000200002. 
Didactic strategies for the development of competences and complex thinking

MORALES, Santiago \& ZAMBRANO, Hugo

2016 Coherencia evaluativa en formación universitaria por competencias: estudio en futuros educadores de Chile. Infancia Imágenes, 15(1), 9-26. https://

MORÍN, Edgar doi.org/10.14483/udistrital.jour.infimg.2016.1.a01.

2003 Introducción al Pensamiento Complejo. Barcelona. Gedisa, S.A.

MURRAY, Gell-Mann

1995 El quark y el jaguar: aventuras en lo simple y lo complejo Barcelona: Editorial Tusquets, Libros para pensar la Ciencia.

OLIVERI, Marie Estance \& MARKLE, Robert

2017 Continuing a culture of evidence: Expanding skills in higher education. ETS Research Report, 1, 1-8. https://doi.org/10.1002/ets2.12137.

ORDÓÑEZ, German, VERDEJO, Peter, CORDERO, Carla María, GUERRA, Verónika, HERNÁNDEZ, Charles, MARTINS, Marco Jesús, MATA, José Lorenzo, MAZÓN, Idalberto, RAICHMAN de MIRASSO, Solier, ROCHA, Juan Alberto, RODRÍGUEZ, Nancy, TOTTER, Ernesto, \& VELASCO, María Inés

2011 Estrategias para el desarrollo de competencias y pensamiento complejo en el aula en asignaturas del área de ingenierías. Universidad Industrial de Santander de Colombia: Innova Censal.

PABLO, Germán

2007 Noción y estructura del dato. Lima: Monografía.

PARDINAS, Joel

2004 Introducción a la Investigación Educacional. México: Panorama Editorial.

PAVIÉ, Almendra

2011 Enfoque basado en competencias: orientaciones sobre sus procedimientos de evaluación. Revista Electrónica de Desarrollo de Competencias (REDEC), 1(7), 114-147.

PEÑA, Johana Carolina

2018 Transformación del Docente desde el Pensamiento Complejo. Revista Scientific, 3(7), 211-230. https://doi.org/10.29394/Scientific.issn.25422987.2018.3.7.11.211-230.

TAPIA, Violeta \& LUNA, Jorge

2010 Validación de una prueba de habilidades de pensamiento para estudiantes de cuarto y quinto de secundaria y primer año de universidad. REVISTA IIPSI, 3(2), 17-59.

TOBÓN, Sergio

2013 Formación integral y competencias. Pensamiento complejo, currículo, didáctica y evaluación. Bogotá: ECOE Ediciones.

TRIGOS, Lina

2001 Pensamiento complejo y competencias en la formación universitaria. Experiencias de innovación en docencia en la Universidad del Rosario. Bogotá: Editorial Universidad del Rosario.

UNIVERSIDAD NACIONAL DE CAÑETE

2016 Estatuto de la Universidad Nacional de Cañete. Cañete: UNDC.

VILLARROEL, Verónica \& BRUNA, Daniela

2019 Do we assess what really matters? The challenge of autenthic assessment in higher education. Revista Calidad de la Educación, 50, 492-509. 
YEOM, Moline

2016 Critical reflection on the massification of higher education in Korea: Consequences for graduate employment and policy issues. Journal of Education and Work, 29(1), 48-63. https://doi.org/10.1080/13639080.2015.1049026.

Fecha de recepción de documento: 15 de diciembre de 2019 Fecha de revisión de documento: 15 de febrero de 2020 Fecha de aprobación de documento: 20 de abril de 2020 Fecha de publicación de documento: 15 de julio de 2020 